This item was submitted to Loughborough's Research Repository by the author.

Items in Figshare are protected by copyright, with all rights reserved, unless otherwise indicated.

\title{
Shear viscosity in hard-sphere and adhesive colloidal suspensions with reverse non-equilibrium molecular dynamics
}

PLEASE CITE THE PUBLISHED VERSION

http://dx.doi.org/10.1039/C7SM00441A

\section{PUBLISHER}

(c) Royal Society of Chemistry

\section{VERSION}

AM (Accepted Manuscript)

\section{PUBLISHER STATEMENT}

This work is made available according to the conditions of the Creative Commons Attribution-NonCommercialNoDerivatives 4.0 International (CC BY-NC-ND 4.0) licence. Full details of this licence are available at: https://creativecommons.org/licenses/by-nc-nd/4.0/

\section{LICENCE}

CC BY-NC-ND 4.0

\section{REPOSITORY RECORD}

Cerbelaud, Manuella, Aleena M. Laganapan, Tapio Ala-Nissila, Riccardo Ferrando, and Arnaud Videcoq. 2019. "Shear Viscosity in Hard-sphere and Adhesive Colloidal Suspensions with Reverse Non-equilibrium Molecular Dynamics". figshare. https://hdl.handle.net/2134/25717. 


\title{
Shear viscosity in hard-sphere and adhesive
}

\section{colloidal suspensions with reverse non-equilibrium molecular dynamics}

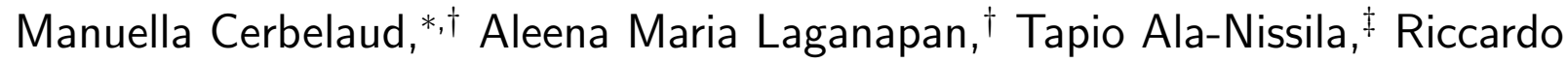 \\ Ferrando, " and Arnaud Videcoq ${ }^{\dagger}$ \\ Univ. Limoges, CNRS, SPCTS, UMR 7315, F-87000 Limoges, France, COMP CoE at the \\ Department of Applied Physics, Aalto University School of Science, P.O. Box 11000, \\ FIN-0076 Aalto, Espoo, Finland, and Department of Physics, Box 1843, Brown University, \\ Providence RI 02912-1843, U.S.A., and Dipartimento di Chimica e Chimica Industriale \\ and CNR-IMEM, via Dodecaneso 31, 16146, Genova, Italy \\ E-mail: manuella.cerbelaud@unilim.fr
}

\begin{abstract}
We employ the reverse non-equilibrium molecular dynamics method (RNEMD) of Müller-Plathe [Phys. Rev. E 59, 4894 (1999)] to calculate the shear viscosity of colloidal suspensions within the stochastic rotation dynamics - molecular dynamics (SRD-MD) simulation method. We examine the influence of different coupling schemes in SRD-MD on the colloidal volume fraction $\phi_{\mathrm{C}}$ dependent viscosity from the dilute

${ }^{*}$ To whom correspondence should be addressed

$\dagger$ Univ. Limoges, CNRS, SPCTS, UMR 7315, F-87000 Limoges, France

${ }^{\ddagger}$ COMP CoE at the Department of Applied Physics, Aalto University School of Science, P.O. Box 11000, FIN-0076 Aalto, Espoo, Finland, and Department of Physics, Box 1843, Brown University, Providence RI

`Dipartimento di Chimica e Chimica Industriale and CNR-IMEM, via Dodecaneso 31, 16146, Genova,
\end{abstract} 02912-1843, U.S.A. Italy 
limit up to $\phi_{\mathrm{C}}=0.3$. Our results demonstrate that the RNEMD method is a robust and reliable method for calculating rheological properties of colloidal suspensions. To obtain quantitatively accurate results beyond the dilute regime, the hydrodynamic interactions between the effective fluid particles in the SRD and the MD colloidal particles must be carefully considered in the coupling scheme. We benchmark the method by comparing with the hard sphere suspension case, and then calculate relative viscosities for colloids with mutually attractive interactions. We show that the viscosity displays a sharp increase at the onset of aggregation of the colloidal particles with increasing volume fraction and attraction.

\section{Introduction}

During the recent years there has been a growing interest in the field of ceramic processing in developing colloidal processes based on additive manufacturing (ink-jet printing, stereolitography etc.). In these processes control on the rheological properties of the suspensions such as the viscosity is crucial to optimize the flow rates in channels and, more generally, to control the final properties of the ceramic objects that are formed. The viscosity and rheological properties are dictated by the spatio-temporal arrangements of the colloids, which depend on the colloidal interactions. ${ }^{1}$

Colloidal suspensions are complex fluids whose behavior is governed by many parameters such as $\mathrm{pH}$, size, and the physical properties of the colloidal particles. Because of these many factors, numerical simulations are a very useful tool in helping to understand and control the suspension properties avoiding expensive experimental trial-and-error procedures. An important point for correctly estimating the rheological properties lies in the correct description

of hydrodynamic interactions in the numerical simulations. ${ }^{1}$ Among the different available numerical methods which model hydrodynamics, the stochastic rotation dynamics coupled with molecular dynamics (SRD-MD) has been proven to be a fast and reliable method for colloidal suspensions. ${ }^{24}$ SRD-MD is a particle based method introduced by Malevanets and 
Kapral in $1999^{5}$ where the fluid is represented by point particles. The dynamics of the fluid is described by the SRD part and the dynamics of the colloids by the MD part which are coupled. Different schemes for coupling can be found in the literature: including the colloids

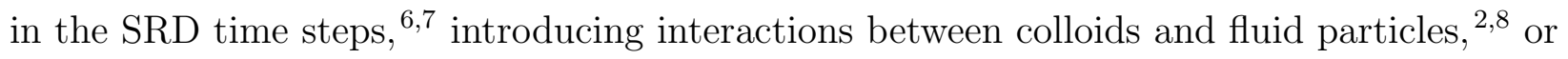
using some collision rules to remove the fluid particles from the colloids and to allow momentum and energy exchange. ${ }^{.79}$ The different schemes do not describe the hydrodynamic interactions at the same level and the choice of the scheme depends on the properties to be studied. 10

In Ref. 11 SRD-MD simulations were used to calculate the shear viscosity of hard sphere suspensions. It was computed by introducing a shear by the Lees-Edwards boundary conditions $^{\sqrt{12}}$ in order to calculate the stress tensor from which the shear viscosity is deduced. This computation method implies introduction of complex boundary conditions as well as the use of a thermostat which can have an uncontrolled influence on the results. Here, we propose an alternate method that does not suffer from such shortcomings. It is based on the idea to calculate the viscosity in SRD-MD simulations by a reverse nonequilibrium molecular dynamics method (RNEMD) proposed by Müller-Plathe. $\frac{13}{13}$ This method reverses the cause and effect picture used in the sense that the momentum is imposed and the velocity gradient is measured. This method is simple because in it only momentum between the particles is exchanged and there is neither the need to use complex boundaries nor to insert a thermostat. In the first part, following Ref. 11, the RNEMD method is used to determine the shear viscosity of hard sphere suspensions. The hard sphere system is an excellent benchmark that allows comparison with many other studies. In our case particular attention is paid to the influence of the different coupling schemes that can be used in SRD-MD to couple the dynamics of the fluid particles and that of the colloids. After benchmarking the method is used to study the shear viscosity of suspensions where colloids interact via an attractive potential, which is a model better suited to study real suspensions where aggregation of the colloidal particles may take place. 


\section{Simulation methods}

\subsection{The hybrid SRD-MD method}

The colloidal system considered in this work is the same as in Refs. 211 and 14. It consists of spherical silica particles of radius $a_{\mathrm{c}}=300 \mathrm{~nm}$ and of mass $M_{\mathrm{c}}=2.49 \times 10^{-16} \mathrm{~kg}$ embedded in water. Density of silica and water are fixed at $\rho_{\mathrm{c}}=2000 \mathrm{~kg} \mathrm{~m}^{-3}$ and $\rho_{\mathrm{f}}=1000 \mathrm{~kg} \mathrm{~m}^{-3}$, respectively. The dynamics of the suspensions is simulated by the hybrid SRD-MD method. In this method the fluid is described by a SRD model with $N_{\mathrm{f}}$ point particles of mass $m_{\mathrm{f}}$. In SRD, the fluid particles evolve according to a simple scheme composed of two steps: a streaming and a collision step. During the streaming step, the position vectors of the fluid particles $\mathbf{r}_{i}$ are updated as follows:

$$
\boldsymbol{r}_{i}\left(t+\Delta t_{\mathrm{SRD}}\right)=\boldsymbol{r}_{i}(t)+\boldsymbol{v}_{i}(t) \Delta t_{\mathrm{SRD}}
$$

where $\boldsymbol{v}_{i}(t)$ is the velocity of particle $i$ at time $t$, and $\Delta t_{\mathrm{SRD}}$ the SRD time step. For the collision step, the simulation box of size $L_{\mathrm{b}}$ is divided in smaller collision cells of size $a_{0}$. For each cell, the collision is performed by rotating the velocities of particles relative to the cell center of mass velocity $\mathbf{v}_{\mathrm{cm}}$ as follows:

$$
\boldsymbol{v}_{i}^{\prime}(t)=\boldsymbol{v}_{\mathrm{cm}}+\boldsymbol{R}(\alpha)\left[\boldsymbol{v}_{i}(t)-\boldsymbol{v}_{\mathrm{cm}}\right]
$$

with $R$ being a stochastic rotation matrix and $\alpha$ the angle of rotation. When the SRD is coupled with MD (SRD-MD), instead of Eq. (1) the fluid particles follow the molecular dynamics equations of motion which are realised with a time step $\Delta t_{\mathrm{MD}}$. In general $\Delta t_{\mathrm{SRD}}>$ $\Delta t_{\mathrm{MD}}$, and therefore several MD steps are performed between two successive SRD collision steps. In SRD, the fluid properties (such as the viscosity) depend on three independent parameters: $\frac{218}{2}$

1. The dimensionless mean free path $\lambda=\Delta t_{\mathrm{SRD}} / t_{0}$, where $t_{0}$ is defined as $t_{0}=a_{0} \sqrt{m_{\mathrm{f}} / k_{\mathrm{B}} T}$ 
( $k_{B}$ and $T$ are the Boltzmann constant and the temperature, respectively). To simulate a liquid $\lambda \ll 1$.

2. The average number of fluid particles per cell $\gamma$. This number has to be kept relatively small in order to have good computational efficiency.

3. The rotation angle $\alpha$ which should be chosen in the range $\left[90^{\circ}, 170^{\circ}\right]$ for a liquid. In this study, all the simulations are performed with $\alpha=90^{\circ}$.

Once the different parameters have been fixed, the temperature is calculated according to the relation

$$
T=\frac{a_{0}^{2} m_{\mathrm{f}}}{k_{\mathrm{B}} t_{0}^{2}}
$$

and in three dimensions the analytical shear viscosity $\eta_{0}$ of the pure fluid can be obtained as the sum of the kinetic and collision contributions as $\frac{15}{15}$

$$
\eta_{0}=\eta_{\text {kin }}+\eta_{\mathrm{col}}
$$

with

$$
\eta_{\text {kin }}=\frac{\gamma k_{\mathrm{B}} T \Delta t_{\mathrm{SRD}}}{a_{0}^{3}}\left[\frac{5 \gamma}{\left(\gamma-1+e^{-\gamma}\right)(4-2 \cos \alpha-2 \cos 2 \alpha)}-\frac{1}{2}\right]
$$

and

$$
\eta_{\mathrm{col}}=\frac{m_{\mathrm{f}}(1-\cos \alpha)}{18 a_{0} \Delta t_{\mathrm{SRD}}}\left(\gamma-1+e^{-\gamma}\right) .
$$

In the hybrid SRD-MD simulations, the dynamics of colloids is described by molecular dynamics (MD). A velocity Verlet algorithm is used to update the positions $\mathbf{r}_{i}$ and the velocities $\mathbf{v}_{i}$ of particles at each time step $\Delta t_{\mathrm{MD}}: \frac{16}{16}$

$$
\boldsymbol{r}_{i}\left(t+\Delta t_{\mathrm{MD}}\right)=\boldsymbol{r}_{i}(t)+\Delta t_{\mathrm{MD}} \boldsymbol{v}_{i}(t)+\Delta t_{\mathrm{MD}}^{2} \frac{\boldsymbol{F}_{i}(t)}{m_{i}}
$$




$$
\boldsymbol{v}_{i}\left(t+\Delta t_{\mathrm{MD}}\right)=\boldsymbol{v}_{i}(t)+\Delta t_{\mathrm{MD}} \frac{\boldsymbol{F}_{i}(t)+\boldsymbol{F}_{i}\left(t+\Delta t_{\mathrm{MD}}\right)}{2 m_{i}},
$$

with forces $\boldsymbol{F}_{i}(t)$ on particle $i$ at time $t$.

In order to model the interaction between the fluid particles and the colloids, the SRD and MD parts have to be coupled. In the present work three different coupling schemes will be analyzed. For each coupling, parameters used in the simulations are chosen in order to reproduce the tracer diffusion coefficient $D_{\mathrm{T}}$ of an isolated silica particle at $T=293 \mathrm{~K}$.

\section{Coupling Scheme I: The velocities of the colloids are rotated during the SRD}

steps $^{\mathbf{6 . 7}}$ A simple way to couple the fluid and the colloid dynamics is to sort the colloidal particles in the SRD cell and include their velocities in the rotation step. For a collision cell with $N_{\mathrm{c}}$ colloids of mass $M_{\mathrm{c}}$, and $N_{\mathrm{f}}$ fluid particles of mass $m_{\mathrm{f}}$, the center of mass velocity of a cell is given by

$$
\boldsymbol{v}_{\mathrm{cm}}(t)=\frac{\sum_{i=1}^{N_{\mathrm{c}}} M_{\mathrm{c}} \boldsymbol{v}_{i}(t)+\sum_{j=1}^{N_{\mathrm{f}}} m_{f} \boldsymbol{v}_{j}(t)}{N_{\mathrm{c}} M_{\mathrm{c}}+N_{\mathrm{f}} m_{\mathrm{f}}}
$$

In this method, the SRD cell size $a_{0}=500 \mathrm{~nm}$ has to be of the order of the colloidal size. This value is chosen as proposed in Ref. 7] such that the cell volume is close to the colloidal volume. The average number of fluid particles per cell is fixed at $\gamma=30$. The mass of the fluid particle is calculated as $m_{\mathrm{f}}=4.167 \times 10^{-18} \mathrm{~kg}$ according to: ${ }^{2}$

$$
m_{\mathrm{f}}=\frac{a_{0}^{3} \rho_{\mathrm{f}}}{\gamma}
$$

The dimensionless mean free path $\lambda$ is fixed at 0.1 . Different values of $t_{0}$ have been tested. Measurements of the tracer diffusion coefficient $D_{\mathrm{T}}$ of an isolated silica particle computed from the mean-square displacement method ${ }^{2177}$ have shown that $t_{0}=3.6 \times 10^{-3} \mathrm{~s}$ is a value which gives the correct result. The SRD time step is thus fixed at $\Delta t_{\mathrm{SRD}}=3.6 \times 10^{-4} \mathrm{~s}$. Simulations are performed in a cubic box of linear size $L_{\mathrm{b}}=20 a_{0}$ and the number of fluid 
particles is

$$
N_{\mathrm{f}}=\frac{\gamma L_{\mathrm{b}}^{3}}{a_{0}^{3}}
$$

For this coupling scheme, the fluid particles are homogeneously spread in the simulation box because they can penetrate the colloids. Because of this the method is unable to faithfully describe all the relevant hydrodynamic interactions in the suspension. For example, lubrication effects ${ }^{[7}$ are completely missing from Coupling Scheme I.

Coupling Scheme II: An interaction potential between the colloids and the fluid particles is introduced in the MD part ${ }^{2 \mid 8}$ In the present work, colloids and fluid particles interact via a repulsive inverse power potential ${ }^{2}$

$$
V_{\mathrm{cf}}= \begin{cases}\epsilon_{\mathrm{cf}}\left(\frac{\sigma_{\mathrm{cf}}}{r}\right)^{12}, & \left(r \leq r_{\mathrm{c}} \equiv 2.5 \sigma_{\mathrm{cf}}\right) \\ 0, & \left(r>r_{\mathrm{c}}\right)\end{cases}
$$

As suggested in Refs. 2 and 8, to avoid depletion attractions $\sigma_{\mathrm{cf}}$ has to be chosen smaller than the colloidal radius $\left(\sigma_{\mathrm{cf}}<a_{\mathrm{c}}\right)$. Under this condition, the fluid particles can slightly penetrate the colloids, which mimics the lubrication forces. The parameters used in this work are the same as those proposed by Tomilov et al.,$^{2}$ i.e. $\sigma_{\mathrm{cf}}=0.8 a_{\mathrm{c}}$ and $\epsilon_{\mathrm{cf}}=2.5 k_{\mathrm{B}} \mathrm{T}$. The SRD parameters are fixed as follows: $a_{0}=a_{\mathrm{c}} / 2=1.5 \times 10^{-7} \mathrm{~m}, \gamma=5$, and $\lambda=0.1$. The mass of a fluid particle is fixed at $m_{\mathrm{f}}=6.75 \times 10^{-19} \mathrm{~kg}$ according to Eq. (10). The time scale $t_{0}$ is determined as proposed in Ref. 2 based on the Stokes-Einstein expression for $D_{\mathrm{T}}$ and the Enskog-Boltzmann-type kinetic theory adapted for slip boundary conditions, which gives $t_{0}=7.37 \times 10^{-4} \mathrm{~s}$. The size of the simulation box is fixed at $L_{\mathrm{b}}=32 a_{0}$ and the number of fluid particles is obtained from: $\underline{211}$

$$
N_{\mathrm{f}}=\frac{\gamma}{a_{0}^{3}}\left(L_{\mathrm{b}}^{3}-N_{\mathrm{C}} \frac{4}{3} \pi \sigma_{\mathrm{cf}}^{3}\right)
$$


Coupling Scheme III: The fluid particles are removed from the colloids at each MD time step which corresponds to stick boundary conditions on the surface of the colloids ${ }^{\mathbf{7 1 9}}$ The third scheme consists of applying given reflection rules during the MD steps if a fluid particle penetrates a colloid. In our work, the method of stochastic reflection proposed by Padding et al.$^{9}$ is applied. This reflection rule is often used because it acts as a thermostat. For this coupling, the angular momentum of the colloid is needed and therefore the colloids' rotational motion is included in the MD step integrations. During an MD step, if a fluid particle overlaps with a colloid it takes half a time step back (-v $\left.\Delta t_{\mathrm{MD}} / 2\right)$ and it is placed on the surface of the colloid at position $\boldsymbol{r}^{*}$ along the shortest vector from the colloid's surface. After this a new velocity $\boldsymbol{v}^{\prime}$ is imposed on this fluid particle. The new velocity $\boldsymbol{v}^{\prime}$ is given by the sum of the local velocity $\boldsymbol{v}_{\text {loc }}$ on the colloid surface and a random velocity component whose normal and tangential components $\boldsymbol{v}_{\mathrm{n}}$ and $\boldsymbol{v}_{\mathrm{t}}$ are taken from the distributions

$$
p\left(v_{\mathrm{n}}\right) \sim v_{\mathrm{n}} \exp \left(-\beta v_{\mathrm{n}}^{2}\right),
$$

and

$$
p\left(v_{\mathrm{t}}\right) \sim \exp \left(-\beta v_{\mathrm{t}}^{2}\right),
$$

with $\beta=m_{\mathrm{f}} /\left(2 k_{\mathrm{B}} T\right)$. The local velocity is defined as

$$
\boldsymbol{v}_{\mathrm{loc}}=\boldsymbol{V}_{i}+\boldsymbol{\Omega}_{i} \times\left(\boldsymbol{r}^{*}-\boldsymbol{R}_{i}\right),
$$

where $\boldsymbol{V}_{i}$ the velocity of the colloid $i, \boldsymbol{\Omega}_{i}$ its angular velocity, and $\boldsymbol{R}_{i}$ its position. When the second half of the time step is completed, the new position is obtained with the velocity $\boldsymbol{v}^{\prime}\left(+\boldsymbol{v}^{\prime} \Delta t_{\mathrm{MD}} / 2\right)$. All the linear momenta and angular momenta changes are added to the colloid: $\Delta \boldsymbol{P}_{i}=\sum \delta \boldsymbol{p}_{j}=\sum m\left(\boldsymbol{v}-\boldsymbol{v}^{\prime}\right)$ and $\Delta \boldsymbol{L}_{i}=\sum m\left(\boldsymbol{r}^{*}-\boldsymbol{R}_{j}\right) \times\left(\boldsymbol{v}-\boldsymbol{v}^{\prime}\right)$. At the end of the time step, the momentum and angular momentum changes are updated for the colloid as $\boldsymbol{V}_{i} \longrightarrow \boldsymbol{V}_{i}+\Delta \boldsymbol{P}_{i} / M_{\mathrm{c}}$ and $\boldsymbol{\Omega}_{i} \longrightarrow \boldsymbol{\Omega}_{i}+\Delta \boldsymbol{L}_{i} / I$, where $I$ is the colloid's momentum of inertia (here $\left.I=(2 / 5) M_{\mathrm{c}} a_{\mathrm{c}}^{2}\right)$. The SRD parameters used for this coupling are the same as for the 
second coupling, namely $a_{0}=a_{\mathrm{c}} / 2=1.5 \times 10^{-7} \mathrm{~m}, \gamma=5, \lambda=0.1$ and $m_{\mathrm{f}}=6.75 \times 10^{-19} \mathrm{~kg}$. The time scale $t_{0}$ is calculated as proposed in Ref. 2 again using the standard Stokes-Einstein expression for $D_{\mathrm{T}}$ and the Enskog friction coefficient for stick boundary conditions: 9

$$
\zeta_{\mathrm{ENS}}^{V}=\frac{8}{3}\left(\frac{2 \pi k_{\mathrm{B}} T m_{\mathrm{f}} M_{\mathrm{c}}}{m_{\mathrm{f}}+M_{\mathrm{c}}}\right)^{0.5} \frac{1}{a_{0}} \gamma \frac{1+2 \chi}{1+\chi}
$$

with $\chi=I /\left(M_{C} a_{\mathrm{c}}^{2}\right)=2 / 5$. Here, $t_{0}=5.177 \times 10^{-4} \mathrm{~s}$ and therefore $\Delta t_{\mathrm{SRD}}=5.177 \times 10^{-5} \mathrm{~s}$. The size of the simulation box is again fixed at $L_{\mathrm{b}}=32 a_{0}$ and the number of fluid particles is given by

$$
N_{\mathrm{f}}=\frac{\gamma}{a_{0}^{3}}\left(L_{\mathrm{b}}^{3}-N_{\mathrm{c}} \frac{4}{3} \pi a_{\mathrm{c}}^{3}\right) .
$$

It is known that this coupling does not strictly correspond to stick boundary conditions. ${ }^{9}$ To obtain them, Lamura's conditions can be applied. This method consists of adding some virtual particles in the solid (here the colloids) to locally increase the viscosity. However in order to not perturb the viscosity, these conditions are not applied and we admit a small amount of slip on the colloid. It is to be noticed that this coupling scheme allows a more precise description of the hydrodynamic radius of the colloids.

Table 1 summarises the different parameters used in the simulations. At the beginning of the runs, colloids and fluid particles are randomly placed in the simulation domain (for the coupling schemes II and III, fluid particles are placed avoiding superposition with colloids). The computational effort increases with the additional interactions such that Scheme II is about 16 times slower than Scheme I, and Scheme III is about 7 times slower than Scheme II.

\section{$2.2 \quad$ Viscosity measurements}

In our previous work the viscosity of hard sphere suspensions was calculated using the

hybrid SRD-MD simulation method. 11 There a shear was introduced and the viscosity was 
Table 1: Parameters used in the hybrid SRD-MD simulations, with $\eta_{0}$ calculated from Eq. (4). In all the Schemes $\lambda=0.1$ and $\alpha=90^{\circ}$.

\begin{tabular}{|c|c|c|c|c|c|c|}
\hline Coupling & $a_{0}(\mathrm{~m})$ & $\gamma$ & $m_{\mathrm{f}}(\mathrm{kg})$ & $t_{0}(\mathrm{~s})$ & $\eta_{0}(\mathrm{Pas})$ & $L_{\mathrm{b}}\left(a_{0}\right)$ \\
\hline Scheme I & $5 \times 10^{-7}$ & 30 & $4.167 \times 10^{-18}$ & $3.6 \times 10^{-3}$ & $3.99 \times 10^{-8}$ & 20 \\
Scheme II & $1.5 \times 10^{-7}$ & 5 & $6.75 \times 10^{-19}$ & $7.37 \times 10^{-4}$ & $1.5 \times 10^{-8}$ & 32 \\
Scheme III & $1.5 \times 10^{-7}$ & 5 & $6.75 \times 10^{-19}$ & $5.177 \times 10^{-4}$ & $2.17 \times 10^{-8}$ & 32 \\
\hline
\end{tabular}

deduced by estimating the stress tensor. In the present study, another method proposed by F. Müller-Plathe is used instead. $\frac{13}{12}$ In this reverse nonequilibrium molecular dynamics method (RNEMD), a momentum flux is imposed whereas the cause (the velocity gradient) is measured. This method is preferred to the method presented in Ref. 11 because it is simple to use and does not require any uncontrollable approximations. Indeed, the RNEMD method only relies on simple exchanges of momenta between the particles, and no thermostat or complicated boundary conditions have to be introduced. The RNEMD method can be summarised as follows:

1. The simulation box is divided in slices along the direction of the shear (here the SRD cells are used along the $y$ direction).

2. In the middle of the box in the $x$ direction, the fluid particle and the colloidal particle with the highest momentum in the $y$ direction are selected.

3. At the bottom of the box in the $x$ direction, the fluid particle and the colloidal particle with the highest momentum in the $-y$ direction are selected.

4. The momenta of the two colloidal particles as well as the momenta between the two fluid particles are exchanged and the amount of momentum $\left(\Delta p_{y}\right)$ transferred between the slices at $y=0$ and $y=L_{\mathrm{b}} / 2$ is calculated. This value is added to $P_{y}$ which is the total momentum transferred in the simulation.

During the simulations, there is a piecewise linear and symmetric velocity profile of $v_{y}(x)$ (see Fig. 1) whose slope gives the value of $\left\langle\left|d v_{y} / d x\right|\right\rangle$. The momentum flux $J_{x}\left(P_{y}\right)$ is then 
calculated as

$$
J_{x}\left(P_{y}\right)=\frac{P_{y}}{2 t A}
$$

where $t$ the simulation time and $A=L_{\mathrm{b}}^{2}$. The shear viscosity $\eta$ can be obtained from

$$
J_{x}\left(P_{y}\right)=-\eta \frac{d v_{y}}{d x}
$$

The exchange of momentum is performed at each SRD step. Data here have been averaged over ten independent simulations. The method has been benchmarked with SRD simulations for a pure fluid. The shear viscosity obtained for the different couplings agrees with the analytical results reported in Table 1 .

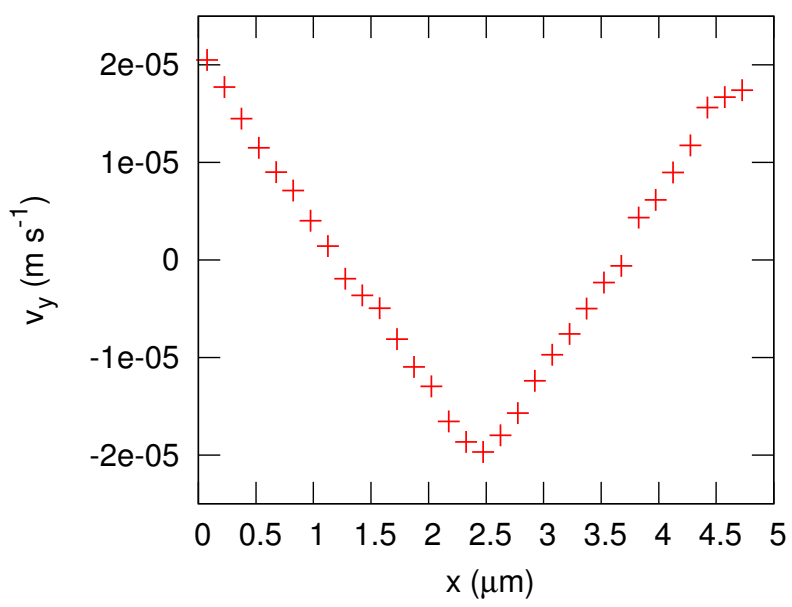

Figure 1: The velocity profile $v_{y}(x)$ obtained from the RNEMD method in a hybrid SRD-MD simulation with coupling scheme II and volume fraction of colloids $\phi_{\mathrm{c}}=0.1$. 


\section{Results and discussion}

\subsection{Shear viscosity of hard sphere suspensions with different cou- pling schemes}

In order to compare the different coupling schemes, benchmark simulations were first performed for hard sphere suspensions. $\frac{18 \sqrt{21}}{2 i f f e r e n t ~ a n a l y t i c a l ~ m o d e l s ~ c a n ~ b e ~ f o u n d ~ t o ~ d e s c r i b e ~}$ the evolution of the relative viscosity of the suspension $\left(\eta_{\mathrm{r}}=\eta / \eta_{0}\right)$ as a function of the colloids' volume fraction $\phi_{\mathrm{c}}{ }^{2{ }^{22}}$ For dilute suspensions, the relative viscosity can be calculated by the Einstein relation, which does not take into account any hydrodynamic effects:

$$
\eta_{\mathrm{r}}=1+2.5 \phi_{\mathrm{c}} .
$$

For semi-dilute suspensions the equation of Batchelor introduces a $\phi_{\mathrm{c}}^{2}$ contribution as

$$
\eta_{\mathrm{r}}=1+2.5 \phi_{\mathrm{c}}+6.2 \phi_{\mathrm{c}}^{2} .
$$

For concentrated suspensions, a semi-empirical formula proposed by Krieger is often used:

$$
\eta_{\mathrm{r}}=\left(1-\frac{\phi_{\mathrm{c}}}{\phi_{\mathrm{m}}}\right)^{-p},
$$

where $\phi_{\mathrm{m}}$ is the maximum packing fraction and $p$ a fitting parameter. In the following, values of $\phi_{\mathrm{m}}=0.74$ and $p=2$ will be used. They give a relatively good approximation to the experimental measurements by van der Werff et al. and Segre et al. $\frac{11118 \mid 19}{\square}$

To describe the interactions between two colloids in the SRD-MD simulations, an inverse 
power law potential adapted to describe hard spheres is used:른

$$
V_{\mathrm{cc}}= \begin{cases}\epsilon_{\mathrm{cc}}\left(\frac{\sigma_{\mathrm{cc}}}{r}\right)^{12}, & \left(r \leq r_{\mathrm{c}} \equiv 2.5 \sigma_{\mathrm{cc}}\right) ; \\ 0, & \left(r>r_{\mathrm{c}}\right),\end{cases}
$$

with $\sigma_{\mathrm{cc}}=2 a_{\mathrm{c}}$ and $\epsilon_{\mathrm{cc}}=2.5 k_{\mathrm{B}} T$. In the following, the volume fraction of the colloids is defined for all the simulations as

$$
\phi_{\mathrm{c}}=\frac{N_{\mathrm{c}} 4 \pi a_{\mathrm{c}}^{3}}{3 L_{\mathrm{b}}^{3}} .
$$

In each simulation run, the shear viscosity $\eta$ is calculated according to the RNEMD method and the relative viscosity $\eta_{\mathrm{r}}$ is obtained by using the corresponding analytical values $\eta_{0}$ for the pure fluid reported in Table 1. The relative viscosities obtained with the different coupling schemes are shown in Fig. 2. The results show that the shear rate $\dot{\gamma}$ differs slightly between the different simulations. It decreases as a function of the volume fraction. The Péclet number defined as $\mathrm{Pe}=6 \pi \eta a^{3} \dot{\gamma} / k_{\mathrm{B}} T$ is in the range $[0.1,0.2],[1.6,2.2]$ and $[1.9,3.1]$ for Coupling Schemes I, II and III, respectively. The shear viscosity is known to depend on the shear rate. In our work, the Péclet numbers are close to each other and thus the results can be compared. Moreover, in the case of hard spheres it has been shown experimentally and numerically that for volume fractions lower than 0.35 , the shear viscosity is almost independent of the shear rate. ${ }^{20}$ According to this, in the following the different viscosities can be considered to correspond to those at zero shear rate.

For the three coupling schemes, the relative viscosity increases with the colloid volume fraction as shown in Fig. 2. For dilute suspensions $\left(\phi_{\mathrm{c}}<0.1\right)$ the results for different schemes are very similar, as expected. At low volume fractions the interparticle hydrodynamic interactions do not play an important role and the Einstein model is a good approximation for $\eta_{\mathrm{r}}$. For volume fractions larger than about 0.1 , we observe that the relative viscosities significantly differ between the coupling schemes. For a fixed $\phi_{\mathrm{c}}, \eta_{\mathrm{r}}$ from Scheme I has the lowest value while from Scheme III it is highest. As we have already mentioned, Scheme 


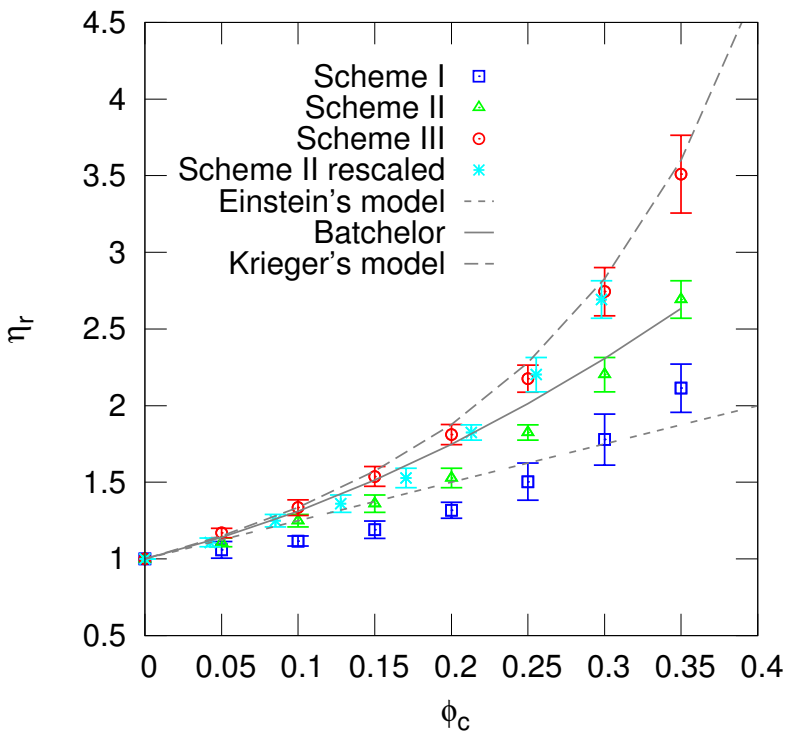

Figure 2: Relative shear viscosity of the suspensions as a function of the colloid volume fraction $\phi_{\mathrm{c}}$ as obtained from SRD-MD with the different coupling schemes. The results are averaged over ten simulations. The Einstein model (Eq. $(20)$ ), the Batchelor formula (Eq.(21)), and the Krieger approximation (Eq. (22p) are also shown. See text for details. 
I cannot correctly describe all the hydrodynamic interactions and lubrication in particular, which is evident in the data beyond the dilute regime. Moreover, for semi-dilute suspensions the relative viscosity is lower than that from Einstein's law, which indicates that the volume fraction is not well defined for this coupling scheme since the colloids are treated as point particles in the SRD cell.

Results obtained with Scheme II are somewhat lower than those from the Krieger law. With this coupling, the radius of a colloid is also not very well defined when interactions between fluid and colloid are considered. The fluid particles can slightly penetrate the colloids and the volume fraction has to be appropriately rescaled. Here we adopt the rescaling from Ref. 2, which consists of converting the volume fractions to a rescaled ones such that the beginning of crystallization corresponds to the theoretical onset of freezing at $\phi_{\mathrm{c}}=0.494$. Following Ref. 2, which considered exactly the same system, the volume fractions were rescaled as $\phi_{\mathrm{c}}^{\mathrm{r}}=(0.494 / 0.58) \phi_{\mathrm{c}}$. The rescaled curve is shown in Fig. 2 with filled blue stars. The rescaled data are in good agreement with the Krieger model indicating good consistency of the rescaling procedure. Finally, for Scheme III the viscosity values are in good agreement with the Krieger model for all volume fractions here without any rescaling. We can thus conclude that the Coupling Scheme III correctly incorporates all the relevant hydrodynamic interactions and is superior to the other schemes described here.

\subsection{Shear viscosity of adhesive colloids}

We will now apply the SRD-MD method with Coupling Scheme III to study the case of adhesive colloids where aggregation is possible due to attractive interactions between the silica particles. We consider a simple model where the silica particles interact via the standard Lennard-Jones (LJ) potential

$$
V_{\mathrm{cc}}=4 \epsilon_{\mathrm{cc}}\left[\left(\frac{\sigma_{\mathrm{cc}}}{r}\right)^{12}-\left(\frac{\sigma_{\mathrm{cc}}}{r}\right)^{6}\right],
$$


with $\sigma_{\mathrm{cc}}=2 a_{\mathrm{c}}$. The parameter $\epsilon_{\mathrm{cc}}$ is varied here to examine different interaction strengths. For semi-dilute suspensions, using a Baxter potential $V_{\mathrm{B}}$ :

$$
V_{\mathrm{B}}= \begin{cases}\infty, & (r<2 a) ; \\ \lim _{\Delta \rightarrow 0} k_{\mathrm{B}} T \ln \left[12 \tau_{\mathrm{B}}\left(\frac{\Delta}{2 a+\Delta}\right)\right], & (2 a \leqslant r \leqslant 2 a+\Delta) ; \\ 0, & (r>2 a+\Delta) .\end{cases}
$$

Cichocki and Felderholf have extended the Batchelor model and they propose that the relative viscosity can be estimated as $\underline{24}$

$$
\eta_{\mathrm{r}}=1+2.5 \phi_{\mathrm{c}}+\left(6.2+\frac{1.9}{\tau_{\mathrm{B}}}\right) \phi_{\mathrm{c}}^{2}
$$

where $\Delta$ is the width of the potential well and $\tau_{\mathrm{B}}$ the Baxter parameter, which characterizes the strength of the attraction between the colloids. The parameter $\tau_{\mathrm{B}}$ tends to zero with increasing attraction, while the hard sphere limit corresponds to $\tau_{\mathrm{B}} \longrightarrow \infty$. According to Eq. (25), the relative viscosity increases with the strength of the attraction for constant volume fraction. Equation (25) has been successfully used to explain the behavior of complex suspensions where the colloids attract each other. $\frac{25}{25}$ Thus, here we will also employ Eq. 25 to analyze our SRD-MD obtained from the LJ potential.

Results from our SRD-MD simulations obtained by changing the well depth $\epsilon_{\mathrm{cc}}$ in the range $\left[0-25 k_{\mathrm{B}} T\right]$ are reported in Fig. 3 for $\phi_{\mathrm{c}}=0.1$. In all the simulations, the Péclet numbers are between 2.3 and 3.3. According to the data, the relative viscosity increases with increasing $\epsilon_{\mathrm{cc}}$. This tendency is in qualitative agreement with Eq. (25). Figure 3 shows also that for $\epsilon_{\mathrm{cc}}<9 k_{\mathrm{B}} T$, the increase in the relative viscosity is rather modest, but beyond this value there is a sharp upturn in $\eta_{\mathrm{r}}$. This change can be correlated to aggregate formation in the simulations. For $\epsilon_{\mathrm{cc}}<9 k_{\mathrm{B}} T$, simulation snapshots in Fig. 4 show that no stable aggregates are formed and the suspensions have a viscosity close to that of the corresponding hard sphere suspension. However, for $\epsilon_{\mathrm{cc}} \geq 9 k_{\mathrm{B}} T$, we can see the formation 
of more stable aggregates which drastically increases the viscosity.

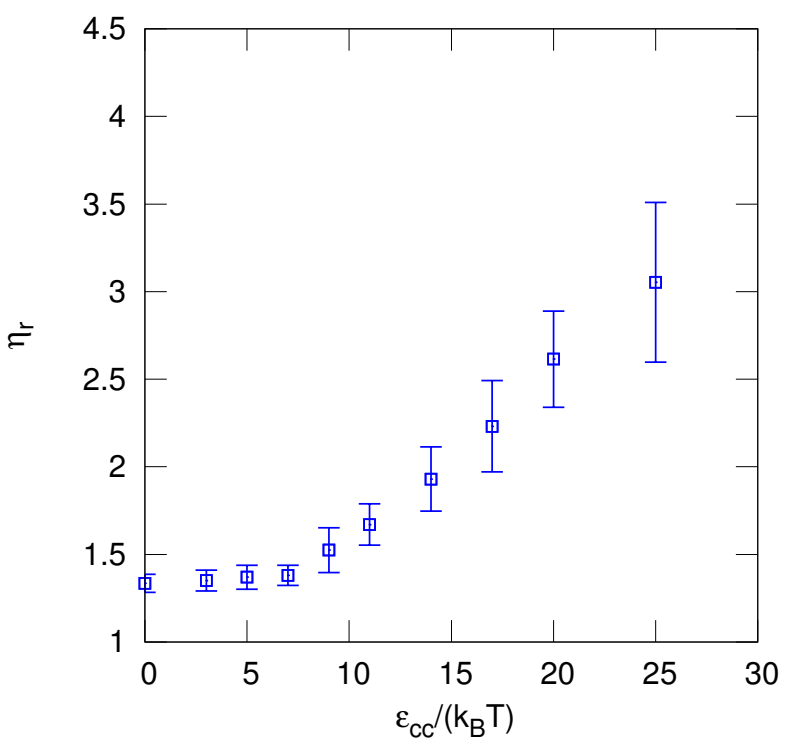

Figure 3: Dependence of the relative viscosity $\eta_{\mathrm{r}}$ on the depth of the attractive well of the LJ potential. Data have been averaged over ten runs. Here $\phi_{\mathrm{c}}=0.1$.

To quantify the picture above we have performed detailed simulations as a function of the volume fraction for two different values of $\epsilon_{\mathrm{cc}}=7 k_{\mathrm{B}} T$ and $14 k_{\mathrm{B}} T$. The results are shown in Fig. 5, where we also show our numerical results for the hard sphere limit with red circles (see Sec. 3.1). The hard sphere data agree well with the Batchelor model up to about $\phi_{\mathrm{c}}=0.2$. For the simulations with $\epsilon_{\mathrm{cc}}=7 k_{\mathrm{B}} T$ and $14 k_{\mathrm{B}} T$, the Péclet number decreases with the volume fraction and is in the range $[1.9,3.1]$ and $[1.4,2.8]$, respectively.

As can be seen in the data, there is good agreement in the semi-dilute regime with Eq. 25), with clear nonlinear dependence of the type $\phi_{\mathrm{c}}^{2}$. Using Eq. 225, the data for $\epsilon_{\mathrm{cc}}=7 k_{\mathrm{B}} T$ and $14 k_{\mathrm{B}} T$ can be fitted using Baxter parameters $\tau_{\mathrm{B}}=0.31$ and $\tau_{\mathrm{B}}=0.05$, respectively.

We note that all the SRD-MD simulations here with Coupling Scheme III are performed with exchanges of momenta between the particles in the RNEMD method for each SRD step. The corresponding frequency $\Gamma 1$ sets the shear rate in the simulations. The relative viscosity is generally given for the zero shear limit. Therefore to examine the effect of the 


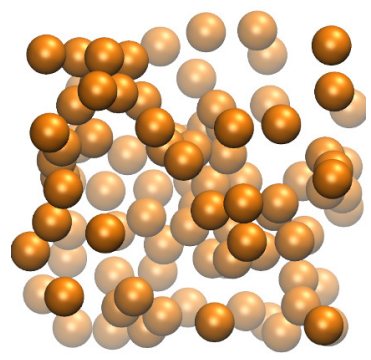

(a)

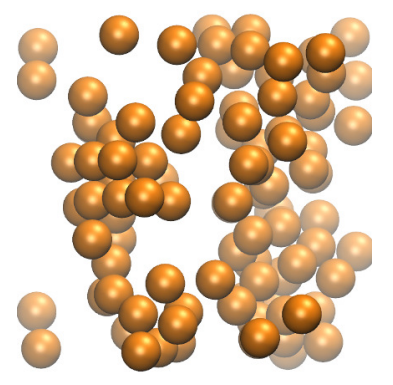

(c)

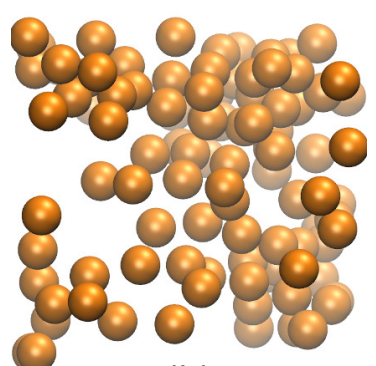

(b)

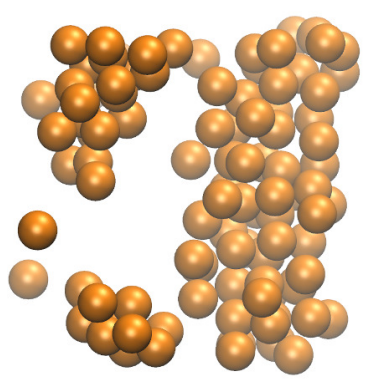

(d)

Figure 4: Snapshots of SRD-MD simulations of attractive colloids with $\phi_{\mathrm{c}}=0.1$ and various LJ well depths: (a) $\epsilon_{\mathrm{cc}}=5 k_{\mathrm{B}} T$, (a) $\epsilon_{\mathrm{cc}}=7 k_{\mathrm{B}} T$, (a) $\epsilon_{\mathrm{cc}}=9 k_{\mathrm{B}} T$, and (d) $\epsilon_{\mathrm{cc}}=11 k_{\mathrm{B}} T$. Aggregates start visibly forming beyond $\epsilon_{\mathrm{cc}} \geq 9 k_{\mathrm{B}} T$ accompanied by a strong increase in the relative viscosity. 


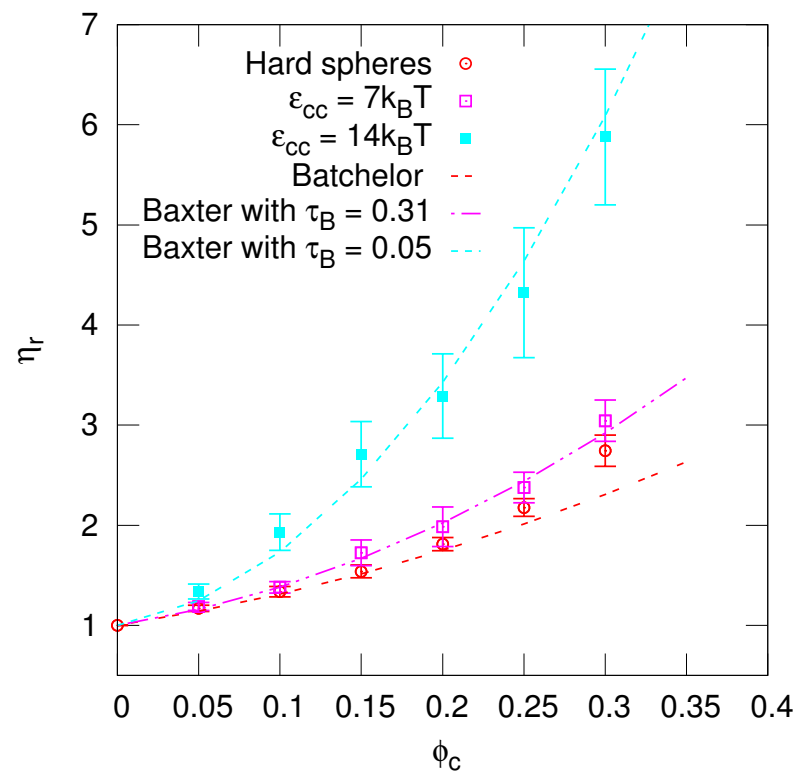

Figure 5: The relative shear viscosity of suspensions with attractive LJ colloids as a function of the colloid volume fraction $\phi_{\mathrm{c}}$ for two values of $\epsilon_{\mathrm{cc}}=7 k_{\mathrm{B}} T$ and $14 k_{\mathrm{B}} T$. The SRD-MD data have been fitted to Eq. (25) with $\tau_{\mathrm{B}}=0.31$ (purple dash-dotted line) and $\tau_{\mathrm{B}}=0.05$ (blue dashed line). For comparison, results obtained for hard spheres are also reported. 
shear rate on the viscosity we performed additional simulations by changing the frequency of the momentum exchanges. Three other frequencies were tested: momenta are exchanged every second SRD step (Г2), every fifth SRD step (Г5), and every tenth SRD step (Г10) $(\Gamma 1>\Gamma 2>\Gamma 5>\Gamma 10)$. The results obtained with $\epsilon_{\mathrm{cc}}=7 k_{\mathrm{B}} T$ are shown in Fig. 6 for $\phi_{\mathrm{c}}=0.1$ and 0.3. A decrease in the frequency induces a decrease in the shear rate and therefore a decrease in the Péclet number. In all the cases, for the same concentration, no clear evolution of the shear viscosity is obtained with Pe. However, when Pe is decreased, the velocity profile becomes very noisy and the results on the shear viscosity are much more imprecise. The data for small Pe are thus somewhat inaccurate due to the increasing role of Brownian motion for small shear rates, and thus $\Gamma 1$ appears here to be a proper rate parameter for the simulations to estimate the viscosity.

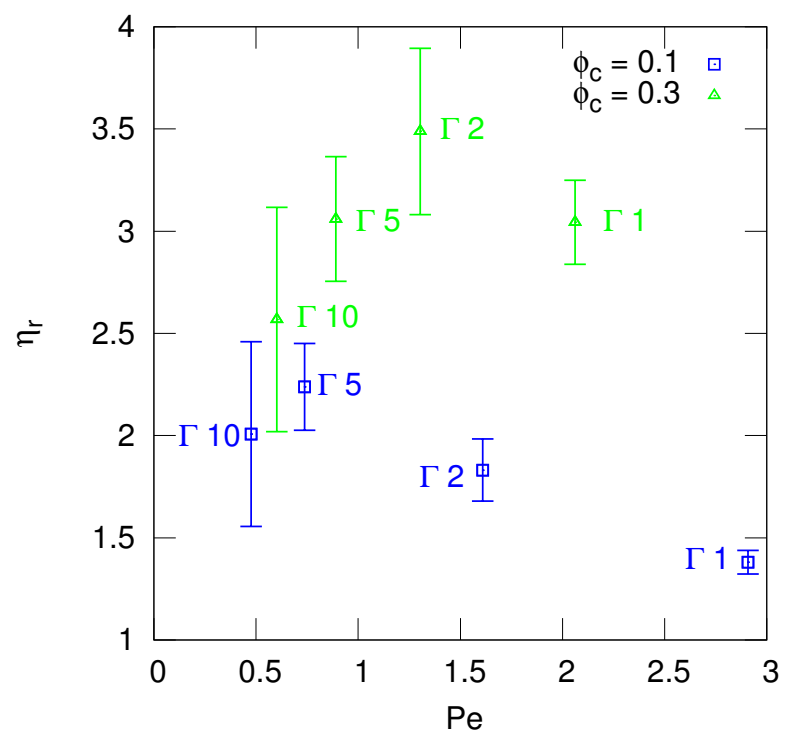

Figure 6: Dependence of the relative viscosity $\eta_{\mathrm{r}}$ on the Péclet number for $\epsilon_{\mathrm{cc}}=7 k_{\mathrm{B}} T$ when the momentum collision rate in the RNEMD method $\Gamma$ is varied for two volume fractions (see text for details). 


\section{Summary and Conclusions}

In this work we have considered the numerical computation of rheological properties of colloidal suspensions and the calculation of the shear viscosity in particular. To this end, we

have employed the reverse non-equilibrium molecular dynamics method of Müller-Plathe $e^{13}$ within the hybrid SRD-MD simulation method, which is an efficient and widely used method for colloidal hydrodynamics. In SRD-MD, there are different options to couple the SRD fluid particles to the MD colloids and we have carefully examined how the coupling scheme influences the volume fraction dependent relative viscosities $\eta_{\mathrm{r}}\left(\phi_{\mathrm{c}}\right)$. In the dilute regime, where hydrodynamic interactions are negligible, results obtained with the different coupling schemes are equivalent. However, for the semi-dilute and concentrated regimes significant differences appear. The coupling used to mimic stick boundary conditions, called Coupling Scheme III here, proves out to be quantitatively accurate to calculate the shear viscosity. In this coupling scheme, hydrodynamic interactions are accurately described and there is no need to rescale the volume fraction. After benchmarking the method by using the hard sphere suspension, we compute the shear viscosity of attractive colloids. Simulations show that the relative viscosity strongly increases at the onset of colloidal aggregation when either the volume fraction of the depth of the attractive potential well is increased. We also find that for semi-dilute suspensions, simulation data agree well with the theoretical model proposed by Cichocki and Felderholf (Eq. 25)). The good agreement obtained here indicates that SRD-MD simulations combined with RNEMD constitute an effective tool to estimate rheological properties and they will be used in the future to determine the viscosity of real ceramic suspensions.

\section{Acknowledgement}

The authors thank CALI and its team for providing the computational facilities (CALI has been financed by the region Limousin, the institutes XLIM, IPAM, GEIST, and the 
University of Limoges). Figure 4 has been obtained by VMD. ${ }^{26}$ T.A-N. has been in part supported by the Academy of Finland through the COMP CoE project numbers 251748 and 284621. R.F. acknowledges support from the University of Limoges for a visiting position.

\section{References}

(1) Brader, J. Nonlinear Rheology of Colloidal Dispersions. J. Phys.: Condens. Matter 2010, 22, 363101.

(2) Tomilov, A.; Videcoq, A.; Chartier, T.; Ala-Nissila, T.; Vattulainen, I. Tacer diffusion in colloidal suspensions under dilute and crowded conditions with hydrodynamic interactions. J. Chem. Phys. 2012, 137, 014503.

(3) Mussawisade, K.; Ripoll, M.; Winkler, R.; Gompper, G. Dynamics of polymers in a particle-based mesoscopic solvent. J. chem. Phys. 2005, 123, 144905.

(4) Watari, N.; Makino, M.; Kikuchi, N.; Larson, R.; Doi, M. Simulations of DNA motion in a microchannel using stochastic rotation dynamics. J. chem. Phys. 2007, 126, 094902.

(5) Malevanets, A.; Kapral, R. Mesoscopic model for solvent dynamics. J. Chem. Phys. $1999, \underline{110}, 8605$.

(6) Winkler, R.; Huang, C.-C. Stress tensors of Multiparticle collision dynamics fluids. J. Chem. Phys. 2009, 130, 074907.

(7) Hecht, M.; Harting, J.; Ihle, T.; Herrmann, H. J. Simulation of claylike colloids. Phys. Rev. E 2005, 72, 011408.

(8) Padding, J. T.; Louis, A. A. Hydrodynamic interactions and Brownian forces in colloidal suspensions: Coarse-graining over time and length scales. Phys. Rev. E 2006, 74, 031402. 
(9) Padding, J. T.; Wysoscki, A.; Löwen, H.; Louis, A. A. Stick boundary conditions and rotational velocity auto-correlation functions for colloidal particles in a coarse-grained representation of the solvent. J. Phys.:Condens. Matter 17 2005, 93, S3393-S3399.

(10) Dahirel, V.; Zhao, X.; Jardat, M. Comparison of different coupling schemes between counterions and charged nanoparticles in multiparticle collision dynamics. Phys. Rev. E 2016, 94, 023317.

(11) Laganapan, A.; Videcoq, A.; Bienia, M.; Ala-Nissila, T.; Bochicchio, D.; Ferrando, R. Computation of shear viscosity of colloidal suspensions by SRD-MD. J. Chem. Phys. 2015, $\underline{142}, 144101$.

(12) Lees, A.; Edwards, S. The computer study of transport processes under extreme conditions. J. Phys. C: Solid State Phys. 1972, 5, 1921-1929.

(13) Müller-Plathe, F. Reversing the perturbation in nonequilibrium molecular dynamics : an easy way to calculate the shear viscosity of fluids. Phys. Rev. E 1999, $\underline{59}, 4894-4898$.

(14) Tomilov, A.; Videcoq, A.; Cerbelaud, M.; Piechowiak, M.; ; Chartier, T.; AlaNissila, T.; Bochicchio, D.; Ferrando, R. Aggregation in colloidal suspensions: evaluation of the role of hydrodynamic interactions by means of numerical simulations. J. Phys. Chem. B 2013, 117, 14509-14517.

(15) Kikuchi, N.; Pooley, C.; Ryder, J.; Yeomans, J. Transport coefficients of a mesoscopic fluid dynamics. J. Chem. Phys. 2003, 119, 6388-6395.

(16) Allen, M. P.; Tildesley, D. J. Computer Simulation of Liquids; Oxford University Press: Oxford, 1987.

(17) Ala-Nissila, T.; Ferrando, R.; Ying, S. C. Collective and single particle diffusion on surfaces. Advances In Physics 2002, 51, 949-1078. 
(18) van der Werff, J.; de Kruif, C. Hard-sphere colloidal dispersions: the scaling of rheological properties with particle size, volume fraction, and shear rate. J. Rheol. 1989, 33, $421-454$.

(19) Segrè, P. N.; Meeker, S.; Pusey, P. N.; Poon, W. C. K. Viscosity and Structural Relaxation in Suspensions of Hard-Sphere Colloids. Phys. Rev. Letters 1995, 75, 958.

(20) Brady, J. Computer simulation of viscous suspensions. Chemical Engineering Science 2001, 56, 2921-2926.

(21) Koelman, J.; Hoogerbrugge, P. Dynamic simulations of hard-sphere suspensions under steady shear. Europhys. Lett. 1993, 21, 363-368.

(22) Quemada, D. Modélisation rhéologique structurelle: dispersions concentrées et fluides complexes; Editions TEC \& DOC - Lavoisier, Paris, 2006.

(23) Falck, E.; Lahtinen, J. M.; Vattulainen, I.; Ala-Nissila, T. Influence of hydrodynamics on many-particle diffusion in 2D colloidal suspensions. Eur. Phys. J. E. 2004, 13, 267275.

(24) Wootersen, A.; Mellema, J.; Blom, C.; de Kruif, C. Linear viscoelasticity in dispersions of adhesive hard spheres. J. Chem. Phys. 1994, 101, 542-552.

(25) Lestriez, B.; Bahri, S.; Sandu, I.; Roué, L.; Guyomard, D. On the binding mechanism of $\mathrm{CMC}$ in Si negative electrodes for Li-ion batteries. Electrochemistry Communications 2007, $\underline{9}, 2801-2806$.

(26) Humphrey, W.; Dalke, A.; Schulten, K. VMD: visual molecular dynamics. J. Molecular Graphics 1996, 14, 33-38. 


\section{Table of contents only}

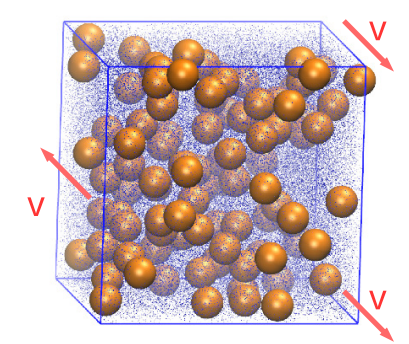

